\begin{tabular}{|c|l|}
\hline Title & Numerical quantification of aerodynamic damping on pitching of vehicle inspired bluff body \\
\hline Author(s) & Cheng, S. Y.; T subokura, M.; Nakashima, T.; Okada, Y.; Nouzawa, T. \\
\hline Citation & $\begin{array}{l}\text { Journal of Fluids and Structures, 30, 188-204 } \\
\text { https:/doi.org/10.1016/.jfluidstructs.2012.01.002 }\end{array}$ \\
\hline Issue Date & 2012-04 \\
\hline Doc URL & http://hdl.handle.net/2115/49228 \\
\hline Type & article (author version) \\
\hline File Information & JFS30_188_204.pdf \\
\hline
\end{tabular}

Instructions for use 


\title{
Numerical quantification of aerodynamic damping on pitching of vehicle- inspired bluff body
}

\author{
S.Y. Cheng ${ }^{a, b}$, M. Tsubokura ${ }^{a, *}$, T. Nakashima ${ }^{c}$, Y. Okada ${ }^{d}$ and T. Nouzawa $^{d}$ \\ ${ }^{a}$ Graduate School of Engineering, Hokkaido University, Kita-13, Nishi-8, Kita-ku, Sapporo, 060-8628, Japan. \\ ${ }^{b}$ Faculty of Mechanical Engineering, Universiti Teknikal Malaysia Melaka, Hang Tuah Jaya, 76100 Durian \\ Tunggal, Melaka, Malaysia. \\ ${ }^{c}$ Graduate School of Engineering, Hiroshima University, 1-4-1 Higashi-Hiroshima, Hiroshima, 739857, Japan. \\ ${ }^{d}$ Vehicle Testing \& Research Department, Mazda Motor Corporation, Aki Gun, Hiroshima, 7308670, Japan. \\ *Corresponding author.Tel. \& Fax: +8111-706-6723,E-mail: mtsubo@eng.hokudai.ac.jp
}

\begin{abstract}
The influence of transient flows on vehicle stability was investigated by large eddy simulation. To consider the dynamic response of a vehicle to real-life transient aerodynamics, a dimensionless parameter that quantifies the amount of aerodynamic damping for vehicle subjects to pitching oscillation is proposed. Two vehicle models with different stability characteristics were created to verify the parameter. For idealized notchback models, underbody has the highest contribution to the total aerodynamic damping, which was up to $69 \%$. However, the difference between the aerodynamic damping of models with distinct A- and C-pillar configurations mainly depends on the trunk-deck contribution. Comparison between dynamically obtained phase-averaged pitching moment with quasisteady values shows totally different aerodynamic behaviors.
\end{abstract}

Keywords: aerodynamic damping; vehicle; transient aerodynamics; pitching; oscillation; LES

\section{Introduction}

Dynamic response of a structure as a result of its interaction with transient flow is of practical interest to many engineering applications. For instance, cable-stayed bridges can experience large amplitude vibration attributed to galloping of dry inclined cables induced at critical Reynolds number (Macdonald and Larose, 2005; Kleissl and Georgakis, 2011), NACA0012 wing can flutter due to flow separation (Poirel et al, 2011), heatexchanger tubes can rupture, and ICI nozzles and guide tubes in a PWR-type nuclear reactor can break by lock-in (Païdoussis, 2006). Moreover, a heavy-lift launch vehicle (such as a space rocket or space shuttle) can respond to buffeting caused by alternate vortex-pair shedding (Dotson and Engblom, 2004). In the case of road vehicles, concern about flow-induced effects is mainly associated with wind noise and vehicle stability. The latter could have more severe consequences because of its impact on drive control and safety.

Typically, the transient flow that affects the attitude of a road vehicle can come from two sources. The first is ambient turbulence such as cross winds (e.g., Baker, 1991a, 1991b, 1991c), gusts of wind (e.g., Tsubokura et al., 2010), and the wake of roadside structures (e.g., Charuvisit et al., 2004) or behind the forward vehicles (e.g., Noger et al., 2005). The second is the airflow generated around the vehicle's body at moderate and high speeds. The size and shape of the airflow structure that forms around a vehicle is determined by the body shape of the vehicle. When the motion of the vehicle is perturbed, which may be caused by disturbances in the road surface or in driver operation, the airflow around it is altered accordingly. In turn, the airflow structure (which changes with vehicle attitude) could induce aerodynamic forces that affect the vehicle's motion. In principle, the airflow and the vehicle body is interacting in a two-way coupling manner. As an example of a study on this phenomenon, Okada et al. (2009) investigated the straight-ahead stability of a sedan-type vehicle with respect to pitching motion, and they reported that the strength of vortices that shed from the sharp-edge A-pillar increases with rear-ride height of the vehicle. As these vortices are convected downstream, they dominate the airflow above the trunk deck. As a result, they strongly influence the characteristic of aerodynamic force exerted on the trunk deck and, consequently, the vehicle's pitching attitude. One way to make the vehicle less susceptible to flow induced effect is by improving its aerodynamic performance so that it becomes less sensitive to wind. Unfortunately, development of vehicle aerodynamics to date has mainly focused on steady-state components, particularly the drag coefficient, $C_{d}$. This 
coefficient can only be used to evaluate performances related to fuel efficiency and top speed; it gives no indication in regard to the vehicle's performance in terms of stability.

A more realistic assessment of a vehicle's aerodynamic performance needs further indicators depending on vehicle type and driving situation under concern. For instance, in the case of high-sided vehicles like vans, buses, and trunks under gusty cross wind, side force, yawing, and rolling moments are important (e.g., Baker and Reynolds, 1992; Sigbjornsson and Snaebjornsson, 1998; Baker et al., 2009). In contrast, in the case of a racing car, down force and pitching moment during cornering are the critical factors (e.g., Katz, 2006; Dominy et al., 2000). Capturing the time-resolved aerodynamic properties is important because steady-state estimation tends to underestimate (or overestimate) the dynamic response. For example, Passmore et al. (2001) investigated the transient aerodynamic effects on a car-like bluff body under sinusoidal side-gust conditions, and they reported that the transient yawmoment response exceeded the quasi-steady value by as much as $30 \%$, while the transient side force was generally lower than the corresponding quasi-steady estimate. Other research carried out on side force and yaw moment (e.g., Tsubokura et al., 2010; Mansor and Passmore, 2007; Macklin et al., 1997; Ryan and Dominy, 1998) has also indicated the incomplete representation of a real transient situation by a steady-state values. Regarding lift coefficient under the influence of ride-height fluctuation, such as that during heaving or pitching, Aschwanden et al. (2006) reported a significant difference in wind-tunnel measurements of a race-car model obtained through steadystate and transient approaches. Moreover, a recent road-test result by Okada et al. (2009) indicates that the straightahead stability behaviors of vehicles with close drag and lift coefficients (differences of only 6.5 and $22.2 \%$, respectively) vary, as shown by their differences in rear-ride height fluctuation and straight-ahead stability sensory ratings. Hence, more and more developments concerning vehicle aerodynamics today no longer rely on a single, steady-state $C_{d}$ but include other parameters that reflect the vehicle's transient aerodynamic characteristics.

The present study investigates the influence of aerodynamic pitching moment on a vehicle's stability. Most studies concerning vehicle stability have focused on crosswind effects. They mainly addressed stability issues related to yawing and rolling. Pitching stability, which has potential impact on drive comfort and handling, particularly at high speed, was often overlooked. The present study therefore aims to address pitching stability of a vehicle. Conventionally, a mechanical suspension is employed to control vehicle attitude. Such a sprung system has been adopted by vehicle design since automobiles were first developed. It has performed satisfactorily in stabilizing vehicle-body attitude; however, the outcome can be augmented by incorporating the transient aerodynamic effects. Recently, Okada et al. (2009) examined the effect of aerodynamics on straight-ahead stability by comparing two passenger cars with identical suspension systems through road tests. They subtly modified some body parts (i.e., styling parameters) of one of the cars to create distinctive aerodynamic features between them. The obtained result was rather astonishing; namely, the difference in rear-ride height fluctuations of the cars was as large as $37 \%$. This result indicates that there is a potential for exploiting flow-induced effects to control vehicle stability. Cheng et al. (2011) investigated pitching stability of notchback-type vehicles and reported that a curved A-pillar configuration has an advantage in obtaining better aerodynamic damping for pitching instability.

Despite the remarkable influence of aerodynamics on vehicle stability, no generalized rating is available for its assessment. Hence, the present study introduces a new index that reflects the dynamic response of a vehicle, in particular, its pitching stability. Similar to $C_{d}$ (which models all the complex dependencies on vehicle drag), the new index is a dimensionless coefficient that considers the complex dependencies of vehicle shape and flow conditions on vehicle stability. This approach predicts the vehicle response by first generating an aerodynamicpitching-moment database obtained through large-eddy simulation (LES) in which the vehicle undergoes forced pitching oscillations in a free stream. The resulted phased-averaged pitching moment is then decomposed into static and dynamic components. The dynamic component (which is in-phase with angular velocity) estimates the amount of damping or enhancement of pitching oscillation by transient aerodynamic effect. Hence, in addition to $C_{d}$, the new coefficient enables a more comprehensive rating for aerodynamic performance of a vehicle. A number of studies have reported that when a vehicle is in motion, quasi-steady analysis is not sufficient owing to the hysteresis effect - that is, the measured aerodynamic force tends to form a loop when plotted against vehicle displacement (e.g., Guilmineau and Chometon, 2008; Aschwanden et al., 2008). Moreover, the degree of aerodynamic hysteresis is proportional to reduced frequency. Discrepancy between quasi-steady and dynamic analyses thus becomes progressively larger with increasing frequency of vehicle motion. Hence, the present study employed a dynamic approach to model the transient aerodynamic properties of vehicles subjected to pitching motion.

\section{Numerical methods}

\subsection{Governing equations}

In the LES, the spatially filtered continuity and Navier-Stokes equations are given as 


$$
\begin{aligned}
& \frac{\partial \bar{u}_{i}}{\partial x_{i}}=0, \\
& \frac{\partial \bar{u}_{i}}{\partial t}+\frac{\partial}{\partial x_{j}} \bar{u}_{i} \bar{u}_{j}=-\frac{\partial \bar{P}}{\partial x_{i}}+2 \frac{\partial}{\partial x_{j}}\left(v+v_{\mathrm{SGS}}\right) \bar{S}_{i j}, \\
& \bar{P}=p / \rho+\left(\overline{u_{i} u_{j}}-\bar{u}_{i} \bar{u}_{j}\right) / 3
\end{aligned}
$$

where $u_{i}, p, \rho$, and $v$ are the $i$-th velocity component, pressure, density, and kinematic viscosity of air. The over-bar indicates a spatially filtered quantity. Strain rate tensor $S_{i j}$ is defined as

$\bar{S}_{i j}=\frac{1}{2}\left(\frac{\partial \bar{u}_{j}}{\partial x_{i}}+\frac{\partial \bar{u}_{i}}{\partial x_{j}}\right)$.

The subgrid-scale (SGS) eddy viscosity, $v_{S G S}$, in Eq. (2) is modeled by the standard Smagorinsky model (Smagorinsky, 1963) as

$$
v_{\mathrm{SGS}}=\left(C_{s} f \Delta\right)^{2} \sqrt{2 \bar{S}_{i j} \bar{S}_{i j}} \text {, }
$$

where $C_{s}$ is the Smagorinsky coefficient, $\Delta$ is the length scale of the SGS turbulence expressed as the cube root of each numerical mesh, and $f_{d}$ represents the damping effect of SGS turbulence in the vicinity of a solid wall. In this study, the Van Driest damping function, $f_{d}$, which is defined as

$$
f_{d}=1-\exp \frac{-y^{+}}{25},
$$

where $y^{+}$is the wall distance, was used.

An in-house CFD code, "FrontFlow/red-Aero," was used for the LES computation. The code was originally developed for the "Frontier Simulation Software for Industrial Science" project. It was then optimized by Tsubokura et al. (2009a) for vehicle-aerodynamics simulation. The code has been validated in previous works; for example, Tsubokura et al. (2009b) showed that numerical results obtained with the code and wind-tunnel measurements of the pressure distribution along the centerline of an ASMO model and of the flow field around a full-scale production car (including a complicated engine room and under-body geometry) agreed well. Interested readers are referred to Tsubokura et al. (2009b) for detailed validations of the code.

\subsection{Discretization}

The governing equations were discretized by using the vertex-centered unstructured finite-volume method. With this method, the governing equations are arranged in the following integral form that describes the conservation of any intensive properties of the flow, $\Phi$ (for mass conservation, $\Phi=1$; for momentum conservation, $\Phi=\boldsymbol{v}$; for conservation of a scalar, $\Phi$ represents the conserved property per unit mass):

$$
\frac{\partial}{\partial t} \int_{\Omega} \rho \Phi \mathrm{d} \Omega+\int_{S} \rho \Phi v \cdot n \mathrm{~d} S=\int_{S} \Gamma \operatorname{grad} \Phi . n \mathrm{~d} S,
$$

where $\Omega$ represents the volume of the control volume (CV), and $S$ represents its surface; the second term on the lefthand side and the term on the right-hand side are convective and diffusion terms, respectively. Each dependent variable was defined on the vertex of the numerical elements, and a virtual CV was constructed around the vertex. Figure 1 shows a simplified two-dimensional graphical illustration of a vertex-centered CV. The governing equations are integrated over the $\mathrm{CV}$. 


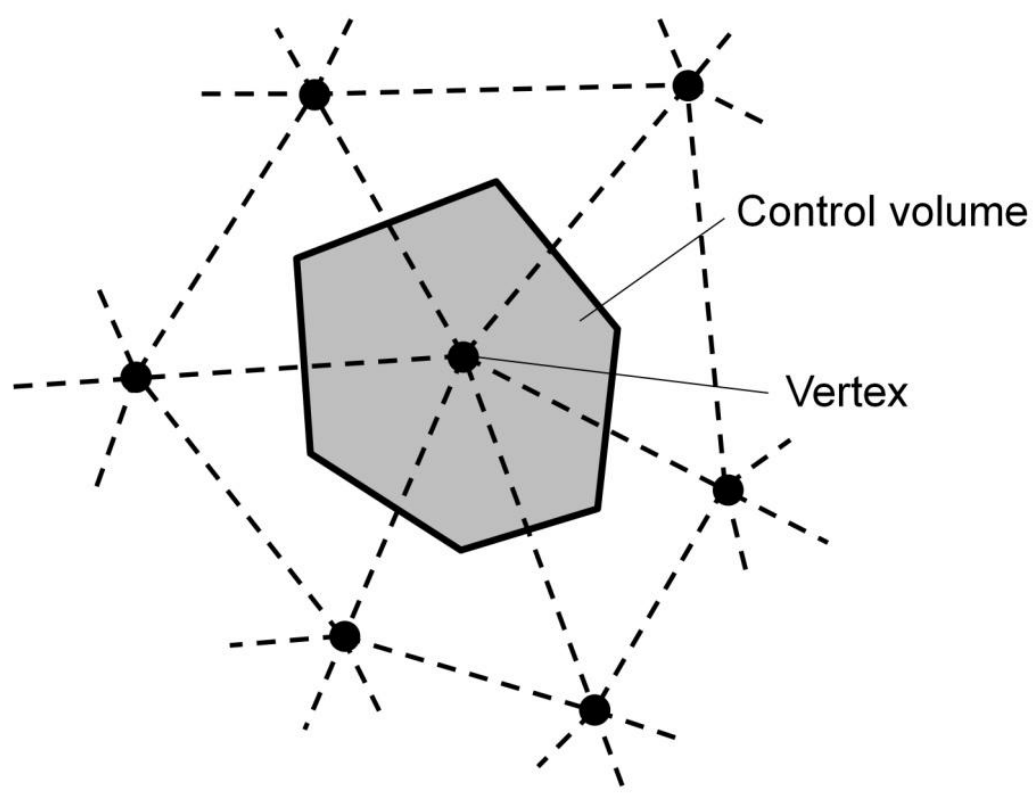

Fig. 1. Vertex-centered control volume.

The second-order central differencing scheme was applied for the spatial derivatives, and blending of a 5\% first-order upwind scheme for the convection term was exploited for numerical stability. Meanwhile, pressurevelocity coupling was preserved by using the SMAC (simplified marker and cell) algorithm by Amsden and Harlow (1970).

For time advancement, Euler implicit method was used. This is because an implicit scheme can accommodate larger time steps than an explicit one, especially in the case of a vehicle simulation in which the velocity and mesh size vary strongly. Hence, the implicit scheme makes the dynamic simulation feasible, especially the one that requires a very long solution time to obtain a reliable phase-averaged statistic. In both the stationary and dynamic LES computations, time difference $\Delta t$ of $1 \times 10^{-5} \mathrm{~s}$ was used.

\subsection{Vehicle models}

Buchheim et al. (1983) indicated that a rounded A-pillar has the advantage of producing lower $C_{d}$ over a sharp-edged configuration. Recently, Okada et al. (2009) revealed that the A-pillar geometry also has remarkable impact on straight-ahead stability of a notchback vehicle. They compared the stability performances of two vehicles, designated vehicle $\mathrm{A}$ and vehicle $\mathrm{B}$, which have significantly different A- and C-pillar configurations. Figure 2 compares the shape of the A- and C-pillars of the vehicles. Relative to vehicle B, vehicle A has a more angular Apillar and a rounder C-pillar. Their road-test results indicate that the vehicle with a rounder A-pillar, i.e., vehicle B, produces relatively lower ride-height fluctuation and scores better in terms of straight-ahead stability rating. It is debatable that the road-test results may also be influenced by the suspension characteristics of the vehicles. However, they ruled out this factor by introducing another vehicle ("modified vehicle A" hereafter) by modifying the body shape of vehicle A on the basis of the geometric features of vehicle B, particularly, a rounded A-pillar styling. The road test with modified vehicle A resulted in a similar trend, namely, lower ride-height fluctuation and better straight-ahead stability rating in comparison with the original version of vehicle $\mathrm{A}$. The reason for the small changes in the shape of A-pillar to affect the ride-height fluctuation is associated with the resulting flow structure above the trunk deck. The more-angular A-pillar in vehicle A generated stronger A-pillar vortices. When convected downstream, these vortices eventually interact with the C-pillar vortices. They formed a more complex flow regime above the trunk deck. Okada et al. (2009) conclude that the complex flow regime has higher lateral fluctuation and induces an unsteady aerodynamic force that promotes the vehicle's vertical motion. On the contrary, flow above the trunk deck of vehicle B (which has weaker A-pillar vortices) was accompanied with little fluctuation, and the resulting aerodynamic force tended to restrain the vehicle's vertical motion. This result confirms the importance of aerodynamics, in additional to mechanical suspension, in vehicle-stability control. 


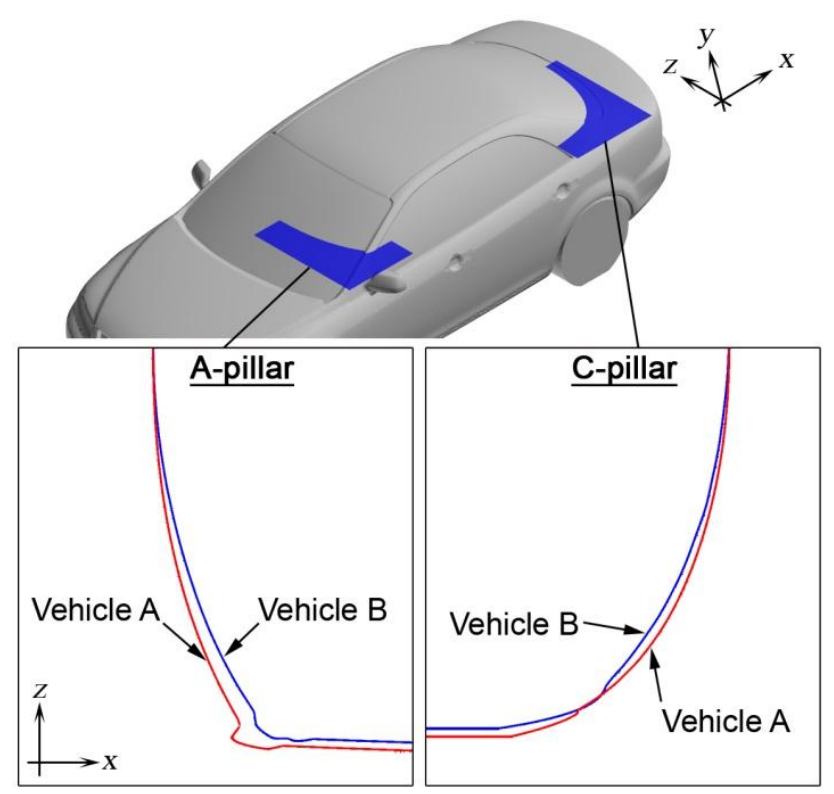

Fig. 2. A- and C-pillar shape configurations of real vehicle models.

To verify the aerodynamic-damping coefficient introduced in this paper, two idealized notchback models were created by taking into consideration the characteristic differences found in the body-shape configurations of real vehicles with distinct stability attitudes. Hence, the model represents the lower-stability notchback was created with a sharp-edged A-pillar and curved C-pillar configurations. In contrast, the other model adopts the opposite configurations (see Fig. 3). Beside these differences, both models have A- and C-pillars with the same slant angles, $30^{\circ}$ and $25^{\circ}$, respectively, which are based on the configurations of a real vehicle. Moreover, the areas of their trunk decks are very similar, namely, about $2.33 \times 10^{-3}$ and $2.31 \times 10^{-3} \mathrm{~m}^{2}$ for models $\mathrm{A}$ and $\mathrm{B}$, respectively. In addition, they comply with a 1:20-scale model with height $h$, width $w$, and length $l$ dimensions of 65, 80, and $210 \mathrm{~mm}$, respectively. For convenience of discussion, the model intended for lower stability is designated "model A", and the one for higher stability as "model B", hereafter.

(a)

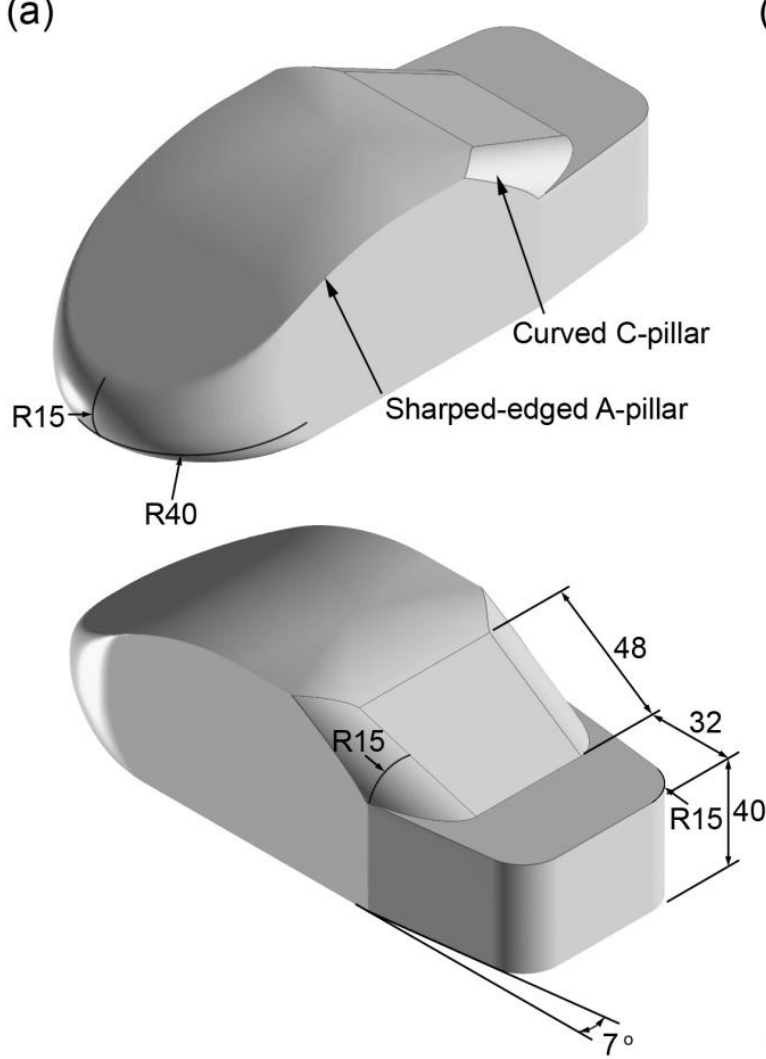

(b)

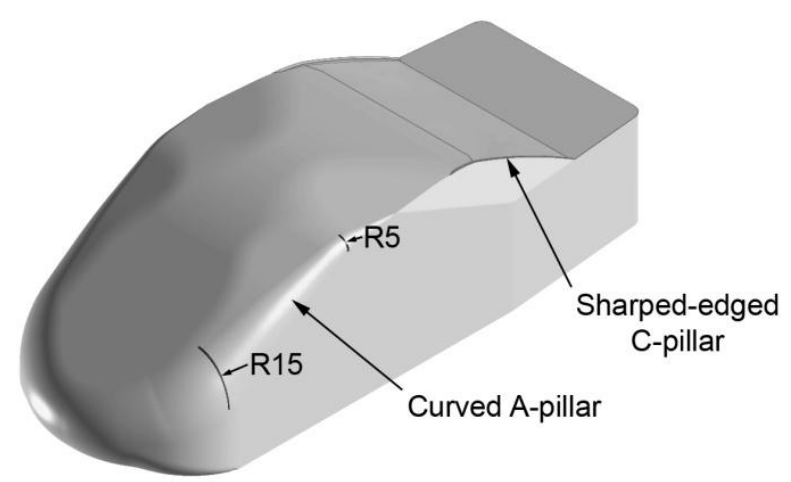

All dimensions in $\mathrm{mm}$

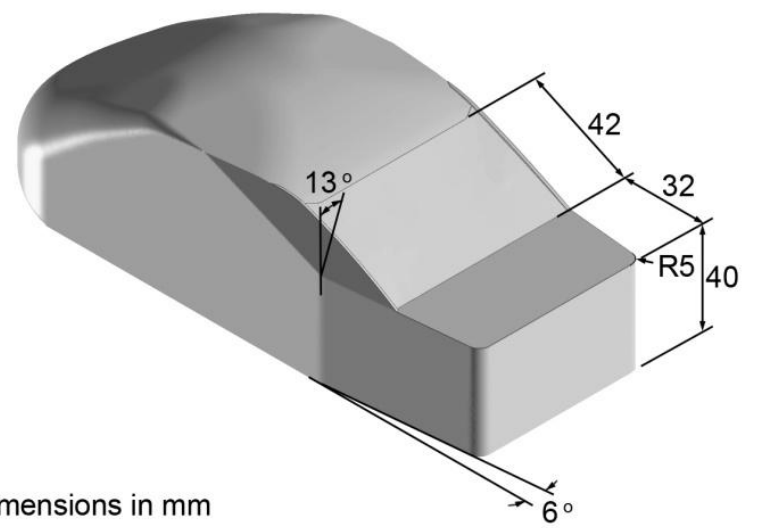

Fig. 3. Simplified vehicle models: (a) Model A; (b) Model B. 


\subsection{Computational domain and boundary conditions}

The computational domain resembles a rectangular wind-tunnel test section. Its cross section covers $1.52 l$ on both sides of the model and height of $2.23 \mathrm{l}$. This set-up produces a small blockage ratio of $1.53 \%$, which is well within the typically accepted range of 5\% in automotive aerodynamic testing (Hucho and Sovran, 1993). The model was situated near the domain floor at a ground clearance of $0.071 l$. The inlet boundary was located $3.14 l$ upstream, while the outlet boundary was $6.86 l$ downstream.

At the inlet boundary, the air flow approaches at a constant velocity of $16.7 \mathrm{~m} / \mathrm{s}$, which corresponds to Re of $2.3 \times 10^{5}$. Meanwhile, a zero-gradient condition is imposed at the outlet boundary. The ground surface is divided into two zones. The upstream zone (which covers $3 l$ from the inlet boundary) is defined as a free-slip wall condition to avoid boundary-layer formation. This setting simulates the wind-tunnel experimental condition in order for consistency in the comparison during validation. The remaining ground is treated with a log-law-based wall function. As for the model surface, the very fine spatial resolution adopted $(y+<4$, as discussed in the next section) makes it possible to estimate the surface friction from the linear velocity profile, which corresponds to the no-slip wall condition. The ceiling and side boundaries of the domain were treated as a free-slip wall-boundary condition.

\subsection{Numerical grids}

The grid topology encompasses several blocks varying in spatial resolution (see Fig. 4). This arrangement provides very fine mesh in regions near the model for capturing more detailed flow information and to use a coarser mesh in other regions for reducing computational effort. Accordingly, the finest block which defined the surface of the model and the boundary layer region is created with fifteen prism mesh layers, with the thickness of the first layer equals to $0.1 \mathrm{~mm}$. As a result, the wall distance of the first-nearest grid point was less than four wall units $\left(y^{+}\right)$. Next to the prism layers is the second finest block, which is intended for capturing important flow structures around the model, especially in the regions near the pillars and truck deck. The third-finest block is located downstream of the model for resolving the larger structures of the decaying wake. Except for the prism mesh, all other blocks are comprised of tetrahedral cells. Overall, the computational domain encompasses around 16 million elements with five million nodes.

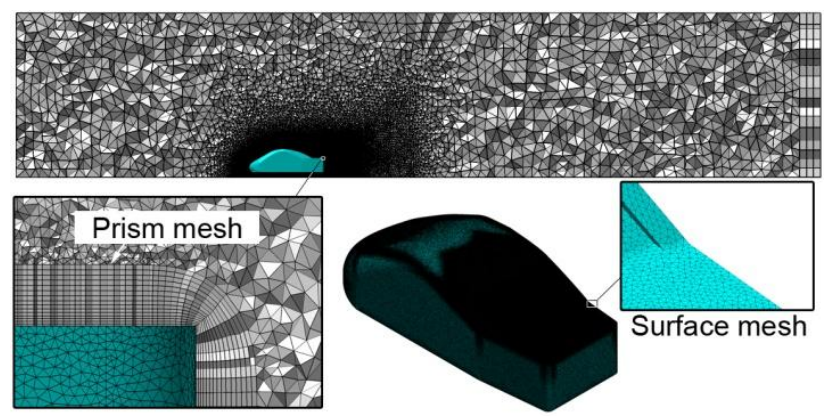

Fig. 4. Computational domain and grid topology.

\subsection{Arbitrary Lagrangian-Eulerian formulation and solution algorithm}

In dynamic simulation cases, motion of the vehicle model causes the nodal points describing the fluid-solid interface to displace relative to the spatial coordinates (i.e., a purely Lagrangian description). To avoid excessive mesh distortion, the surrounding mesh is deformed in an arbitrary Lagrangian-Eulerian (ALE) manner (Hirt et al., 1974), which is calculated using a spring-analogy method. The mesh deformation generates a mass flux on $S$, defined as $-\rho u_{g} S$, where $u_{g}$ is the deformation speed of $S$ in its normal direction; (The negative sign appears by virtue that the flux is generated in the opposite direction to the mesh motion.) $u_{g}$ is defined as $u_{g}=\boldsymbol{u}_{d} \boldsymbol{n}_{s}$, where $\boldsymbol{u}_{d}$ is the deformation velocity vector of the center of $S$, which is evaluated by first-order time difference of its deformed distance during the time step; and $\boldsymbol{n}_{s}$ is an outward unit normal to $S$.

Subsequently, the mass flux is taken into account in the convection terms in the governing equations. First, the convection term in Eq. (2) becomes

$$
\frac{\partial}{\partial x_{j}} \bar{u}_{j}\left(\bar{u}_{j}-\bar{u}_{d, j}\right) \text {. }
$$

And the pressure-Poisson equation derived by enforcing the continuity constraint Eq. (1) through fractional-step method becomes 
$\frac{\partial^{2} \bar{p}}{\partial x_{i}^{2}}=\frac{1}{\Delta t}\left[\frac{\partial\left(\bar{u}_{i}-\bar{u}_{d, i}\right)}{\partial x_{i}}\right]$.

Accordingly, in each new time step, the fluid-solid-interface points move to new positions defined by the imposed sinusoidal function, and the whole mesh is displaced in a fashion similar to the deformation of an elastic body. The corresponding velocity for each $\mathrm{CV}$ surface is then computed and inserted into the related convective terms. Finally, solutions to all the governing equations are obtained exactly like those in the case of a fixed-grid simulation.

\subsection{Verification and validation}

\subsubsection{Grid-convergence study}

To verify that the LES solution is independent of grid size, a grid-refinement study using the generalized Richardson extrapolation method presented by Roache (1998) was conducted. This method makes it possible to determine the error band in the LES result obtained from the chosen grid resolution. Error band $G C I$, which indicates how far the respective solution of a given grid is from the extrapolated asymptotic value (i.e., the actual value as the grid resolution approaches zero), is defined as

$$
G C I_{i-1, i}=\frac{F s\left|\left(g_{i-1}-g_{i}\right) / g_{i}\right|}{\left(r^{\gamma}-1\right)},
$$

where $g_{i}$ is solution obtained from a particular grid scheme ( $i=1,2$, and 3 correspond to finest, medium, and coarsest grids, respectively), $F s$ is a safety factor, which equals 1.25 (for comparison over three or more grids), $r$ is refinement ratio, and $\gamma$ is order of convergence defined as

$$
\gamma=\ln \left(\frac{g_{3}-g_{2}}{g_{2}-g_{1}}\right) / \ln (r) \text {. }
$$

In this study, three grid schemes were created by using the same $r$ of 1.5. Grid resolution is controlled by two parameters: thickness of the prism mesh layers (which determines the grid spacing normal to the vehicle surface) and nodal spacing at each zonal interface.

The main objective of the LES is to determine the aerodynamic pitching moment $M$, exerted on the vehicle models. Table 1 summarizes the grid information and the resulting $\langle M\rangle_{t}$ computed from the three grid schemes as well as the corresponding error bands. Each solution is a time-averaged value over physical time of $0.3 \mathrm{~s}$, which corresponds to about $24 l$ and 30,000 steps. The column "normalized grid spacing" is the spacing normalized by the spacing of the finest grid. The distribution of $\left\langle C_{p \text { stat }}\right\rangle_{t}$ and the streamwise velocity component (normalized by inlet velocity) in the symmetry plane of the model are shown in Figures 5 and 6, respectively. An appreciable difference between the solutions obtained with the coarsest grid and the two finer grids is in the wake behind the rear-shield. The closed-up view in Fig. 7 reveals that the discrepancy between the coarsest-grid solution and the finer-grid solutions was caused by the delay of flow separation from the roof. In consideration of the excessive amount of time required in the dynamic simulations, the medium grid scheme was adopted. For a detailed procedure of the gridconvergence study which may be adopted for CFD computation using an unstructured grid, the reader is referred to Cheng et al. (2011).

Table 1. Grid schemes and the corresponding $\langle M\rangle_{t}$ and error band.

$\begin{array}{llllll}\text { Grid scheme } & \begin{array}{l}\text { 1st wall distance } \\ {[\mathrm{mm}]}\end{array} & \text { Numerical cell\# } & \text { Normalized grid spacing } & \begin{array}{l}\langle M\rangle_{t} \\ {[\mathrm{Nm}]}\end{array} & \begin{array}{l}\text { Error band } \\ {[\%]}\end{array} \\ \text { Finest } & 0.067 & 23,679,780 & 1 & 0.01024 & 0.64 \\ \text { Medium } & 0.1 & 16,073,811 & 1.5 & 0.009802 & 5.97 \\ \text { Coarsest } & 0.15 & 8,065,881 & 2.24 & 0.005747 & 61.45\end{array}$


(a)

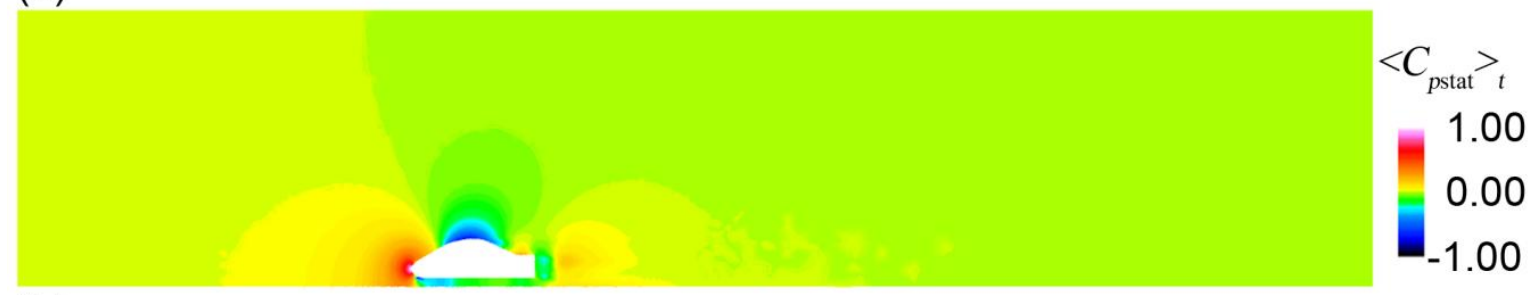

(b)

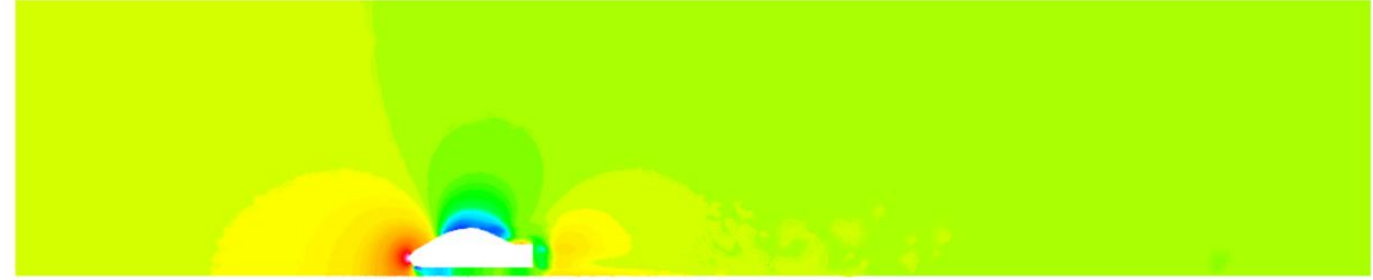

(c)

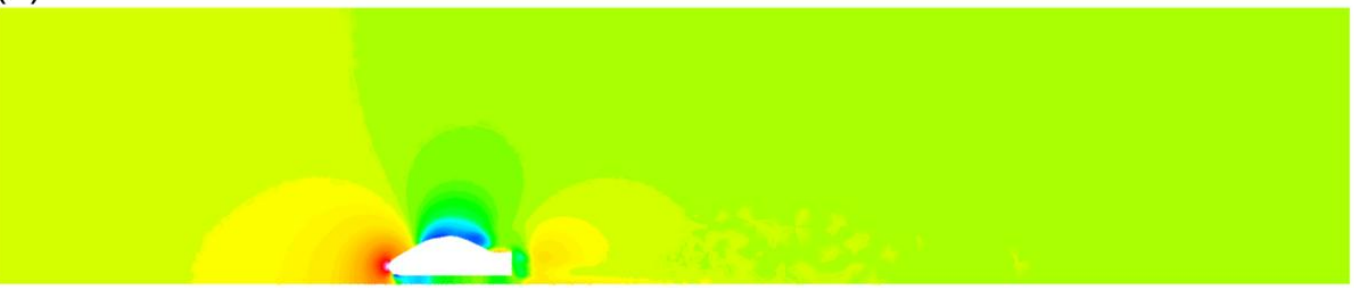

Fig. 5. Static-pressure distribution in the symmetry plane obtained with three grid schemes: (a) coarsest, (b) medium, and (c) finest.

(a)

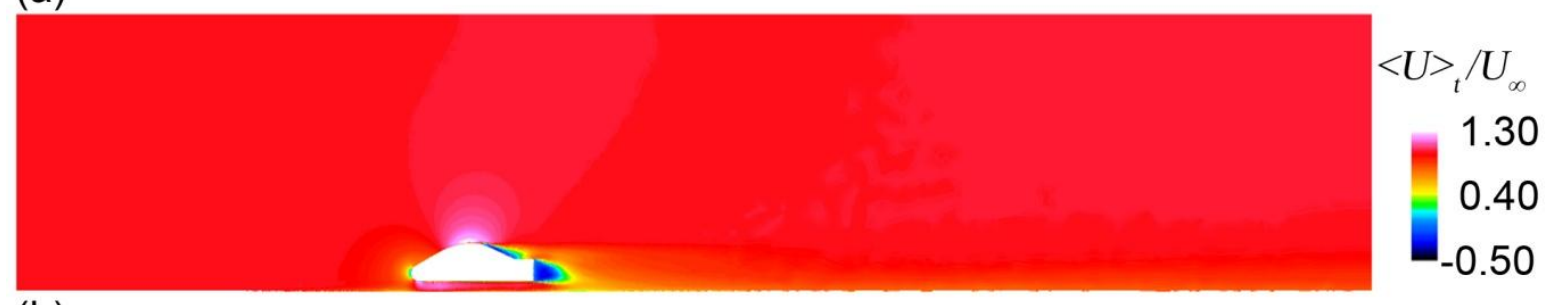

(b)

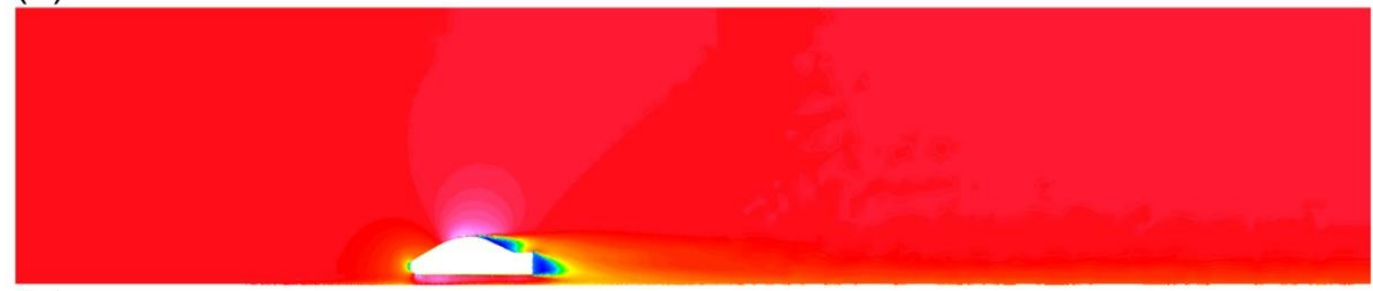

(c)

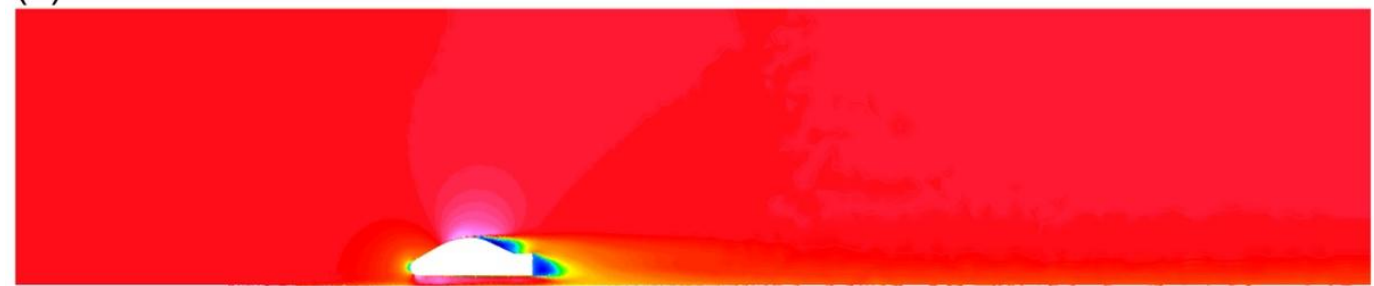

Fig. 6. Streamwise velocity-component distribution in the symmetry plane obtained with three grid schemes: (a) coarsest, (b) medium, and (c) finest. 
(a)

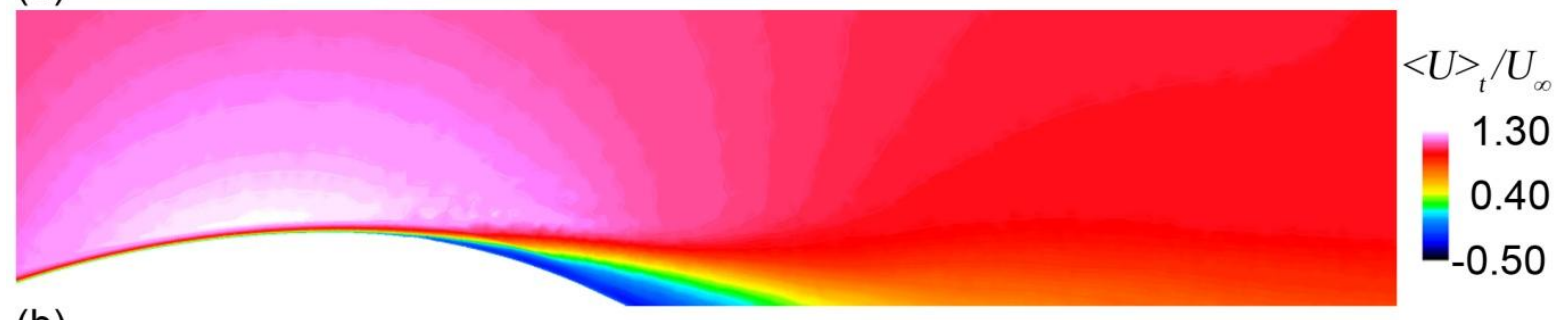

(b)

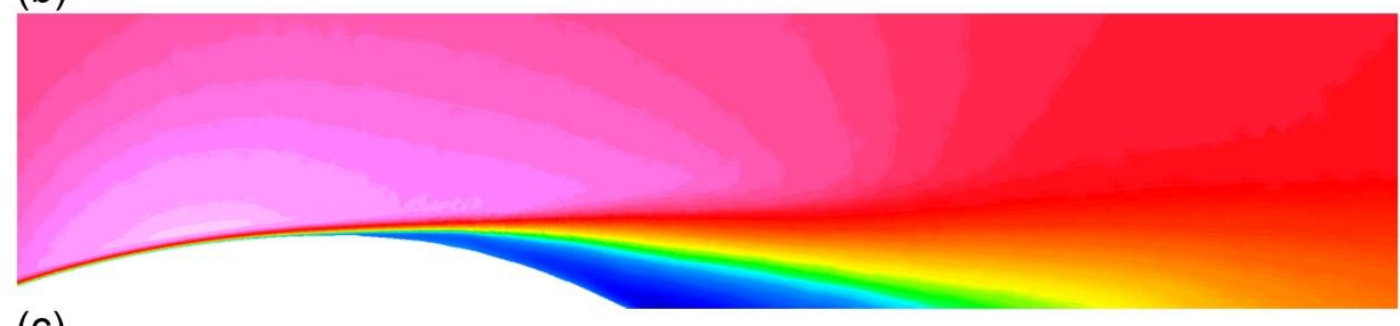

(c)

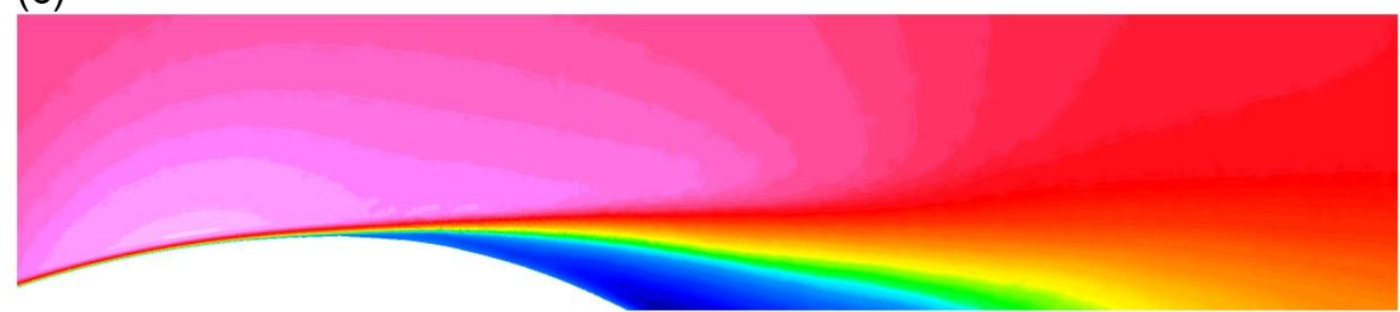

Fig. 7. Closed-up view of streamwise velocity-component distribution at the roof of the model obtained with three grid schemes: (a) coarsest, (b) medium, and (c) finest.

\subsubsection{Optimization of Smagorinsky coefficient}

In the literature, different optimized values have been proposed for the Smagorinsky coefficient, $C_{s}$, for different types of flow field, e.g., $C_{s}=0.1$ for channel flow, and $C_{s}=0.23$ for isotropic decay flow. In the case of a bluff body, which a road vehicle is categorized as, commonly applied values may range from 0.1 to 0.15 . To choose an optimum $C_{s}$ setting, two sets of results obtained with $C_{s}=0.1$ and 0.15 were compared (see Fig. 8). The comparisons show no significant difference between the distribution of time-averaged static pressure coefficient $\left\langle C_{p \text { stat }}\right\rangle_{t}$ along the centerline of model A. Moreover, the percentage difference in time-averaged drag $\langle D\rangle_{t}$, lift $\langle L\rangle_{t}$, and pitching moment $\langle M\rangle_{t}$, given in Table 2 , is only about $0.6,1.8$, and $2.1 \%$, respectively. In consideration that the unstructured mesh used for the numerical grids might affect a certain level of numerical oscillation owing to mesh non-orthogonality, a $C_{s}$ value of 0.15 was therefore adopted. Though small, the artificial dissipation introduced by a higher $C_{s}$ value may offset the numerical oscillation issue.

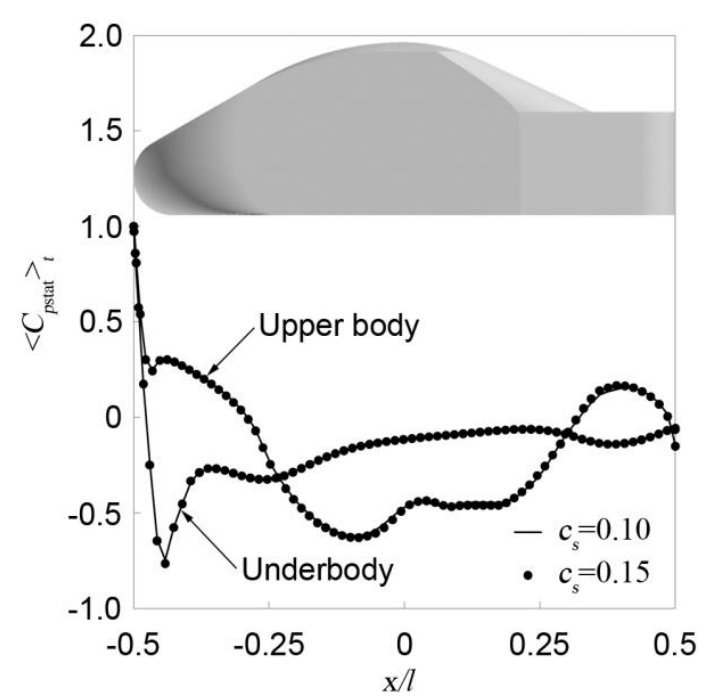

Fig. 8. Static pressure distribution along model A centerline at $C_{s}=0.10$ and 0.15 . 
Table 2. Aerodynamic forces and pitching moment at $C_{s}=0.10$ and 0.15 .

\subsubsection{Comparison with wind-tunnel data}

To validate the numerical method, measured total pressure distribution around the models and LESobtained quantities were compared. The measurements were conducted in the Mazda Wind Tunnel, which has a closed test section with cross-sectional area of $6 \times 4 \mathrm{~m}$ and a length of $12 \mathrm{~m}$. The scale of the models is quite small with respect to the size of the wind tunnel, so the models were mounted on a $1 \times 2 \mathrm{~m}$ acrylic sheet elevated $0.71 \mathrm{~m}$ from the tunnel floor to avoid the boundary-layer effect. It should be noted that the models used in the stationary simulations were supported by four stilts to match the wind tunnel setup. However, to allow pitching oscillation, the stilts were excluded in the dynamic simulations. For consistency of comparison, both the LES and the wind-tunnel measurements were conducted at the same Reynolds number.

Figure 9 compares the distributions of the time-averaged total pressure coefficients obtained by the LES and the wind-tunnel experiment. The visualization planes were at three different streamwise locations, as illustrated in the figure. At $x / l=-0.5$, both the experiment and LES results show a drop in total pressure in the upper-side flow region of model A (marked "I"). This region coincides with the A-pillar vortex location depicted in Fig. 4; it thus implies that the total pressure drop is caused by the A-pillar vortex. Further downstream, the magnitude of the total pressure drop that corresponds to the A-pillar vortex decreases, while its region expands. This result indicates that the Apillar vortex is decaying as it is convected downstream. Meanwhile, the experiment and LES results have also shown the total pressure drop caused by the C-pillar vortex, circulatory structure, and wake of the two models, marked "II," "III," and "IV," respectively. Despite some discrepancy, the LES result is generally in good qualitative agreement with the wind-tunnel measurements. 

$\left\langle C_{\text {poot }}\right\rangle_{\text {, }}$
ㅁ.1-1.2
ㅁ1-1.1
口0.9-1
$\square 0.8-0.9$
$\square 0.7-0.8$
$\square 0.6-0.7$
- $0.5-0.6$
- $0.4-0.5$
- $0.3-0.4$
- $0.2-0.3$
- 0.1-0.2
- $0-0.1$
- $-0.1-0$
- $-0.2--0.1$

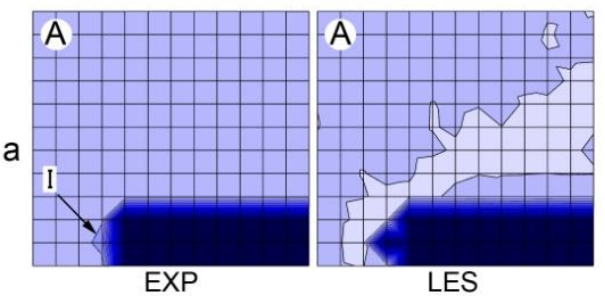

LES

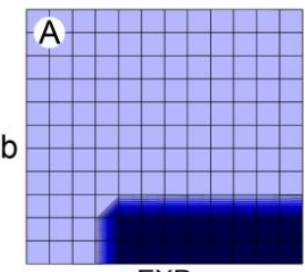

EXP

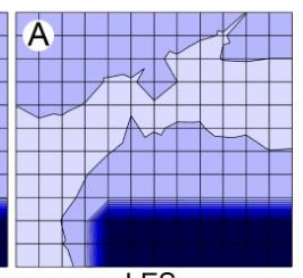

LES

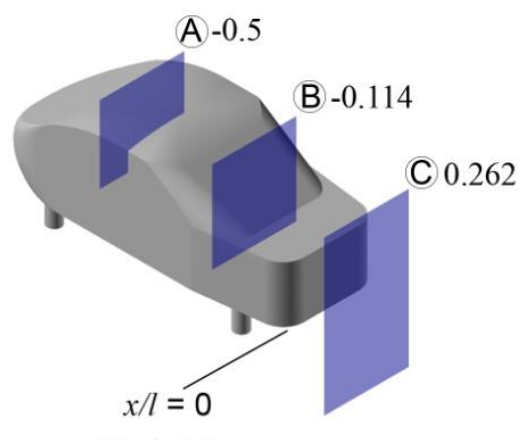

Model A

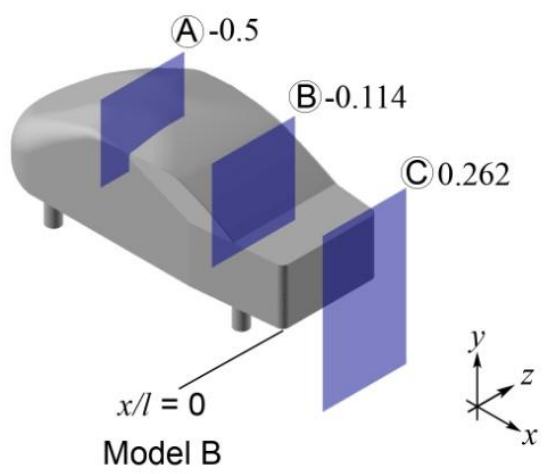

Model B

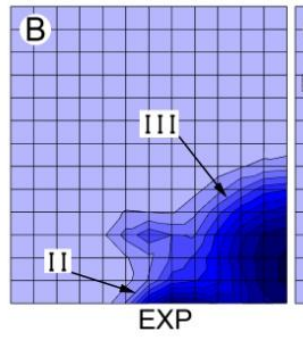

EXP

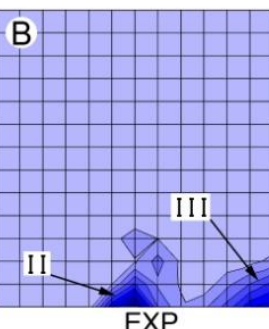

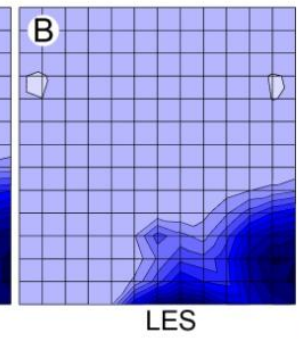

LES

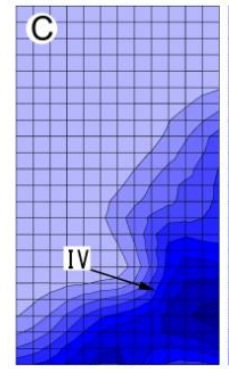

EXP

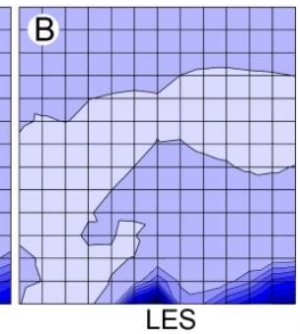

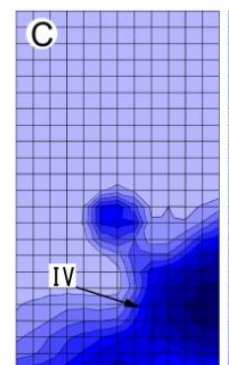

EXP

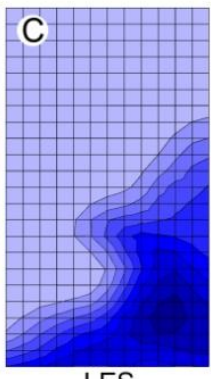

LES

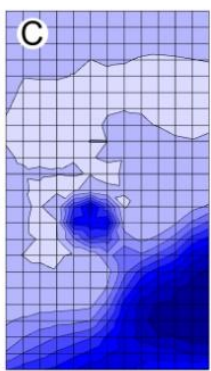

LES

Fig. 9. Comparison of total pressure distribution between wind-tunnel measurement and LES: (a) Model A; (b) Model B.

\section{Results}

\subsection{Verification of simplified vehicle model}

To ensure that the models successfully reproduce the flow features of real vehicles with distinct stability characteristics, flow visualization by LES was conducted, and the flow fields around the simplified models were compared with those of the real vehicles used in the study by Okada et al. (2009). Figures 10 and 11 show the timeaveraged total pressure coefficient, $\left\langle C_{p \text { tot }}\right\rangle_{t}$ (top), cross-flow velocity vectors (middle), and streamlines (bottom) around the real vehicles and simplified models, respectively. In the case of vehicle A, an outward rotating structure, see Fig. 10 (middle), sheds from the A-pillar, and then propagates downstream along the roof side (indicated by the $\left\langle C_{p \text { tot }}\right\rangle_{t}$ contour and the streamlines). At the rear windshield, the trajectory bends inward and continues downstream above the windshield and trunk deck. This structure is termed a "A-pillar vortex" and can also be found in vehicle B, though it is weaker owing to the smoother A-pillar configuration (see Fig. 1). Meanwhile, there is a rotational structure (i.e., vortex) that rolls up along the C-pillar of the two vehicles and then convected downstream along the side of the trunk deck. However, $\left\langle C_{p \text { tot }}\right\rangle_{t}$ in Fig. 10 (top) shows that the structure, namely, the C-pillar vortex, is more intense and concentrated in vehicle B owing to the more angular C-pillar configuration (see Fig. 1). 
(a)

(b)
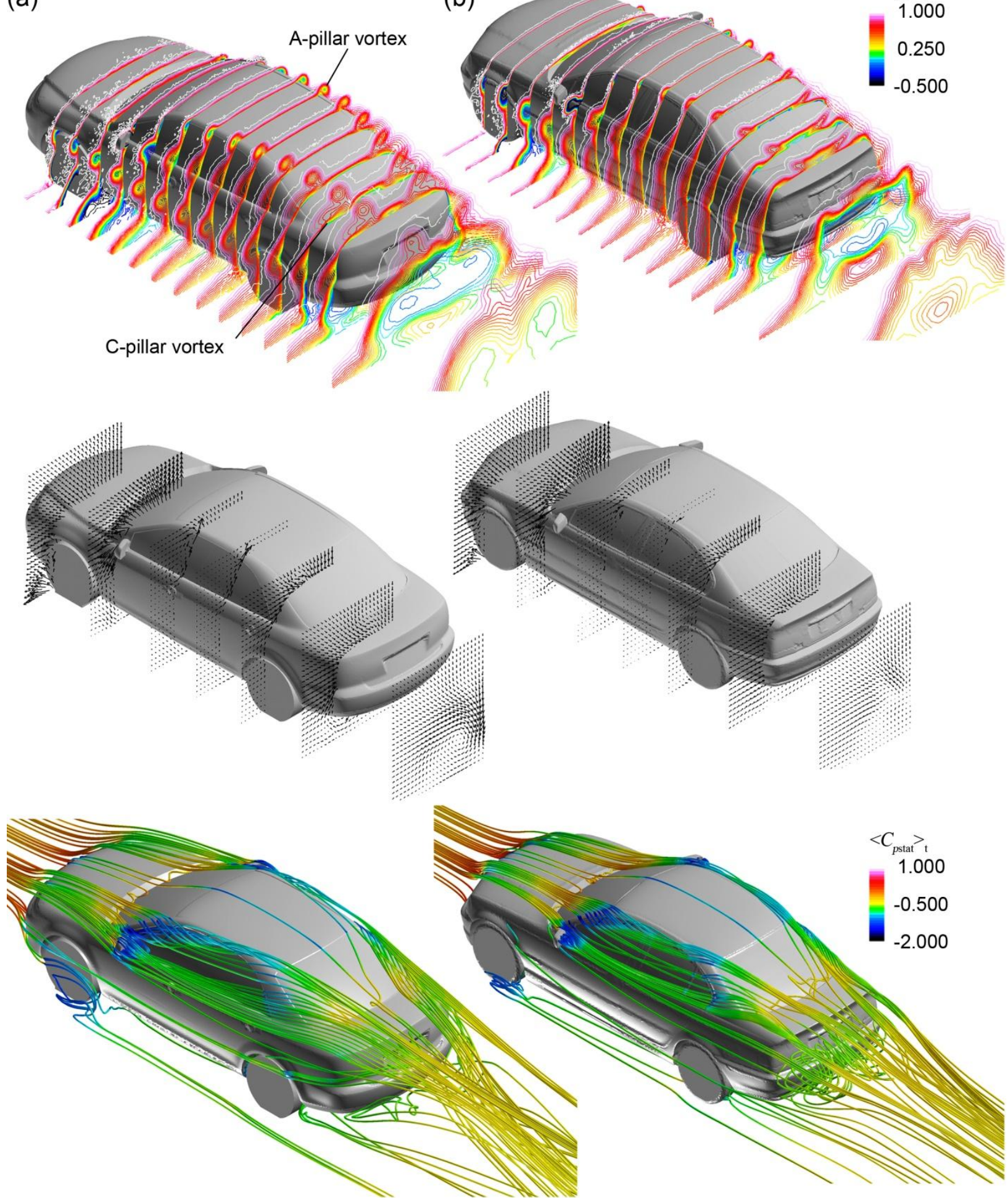

Fig. 10. Predominant flow structures around real vehicles: (a) Vehicle A; (b) Vehicle B. 
(a)

(b)
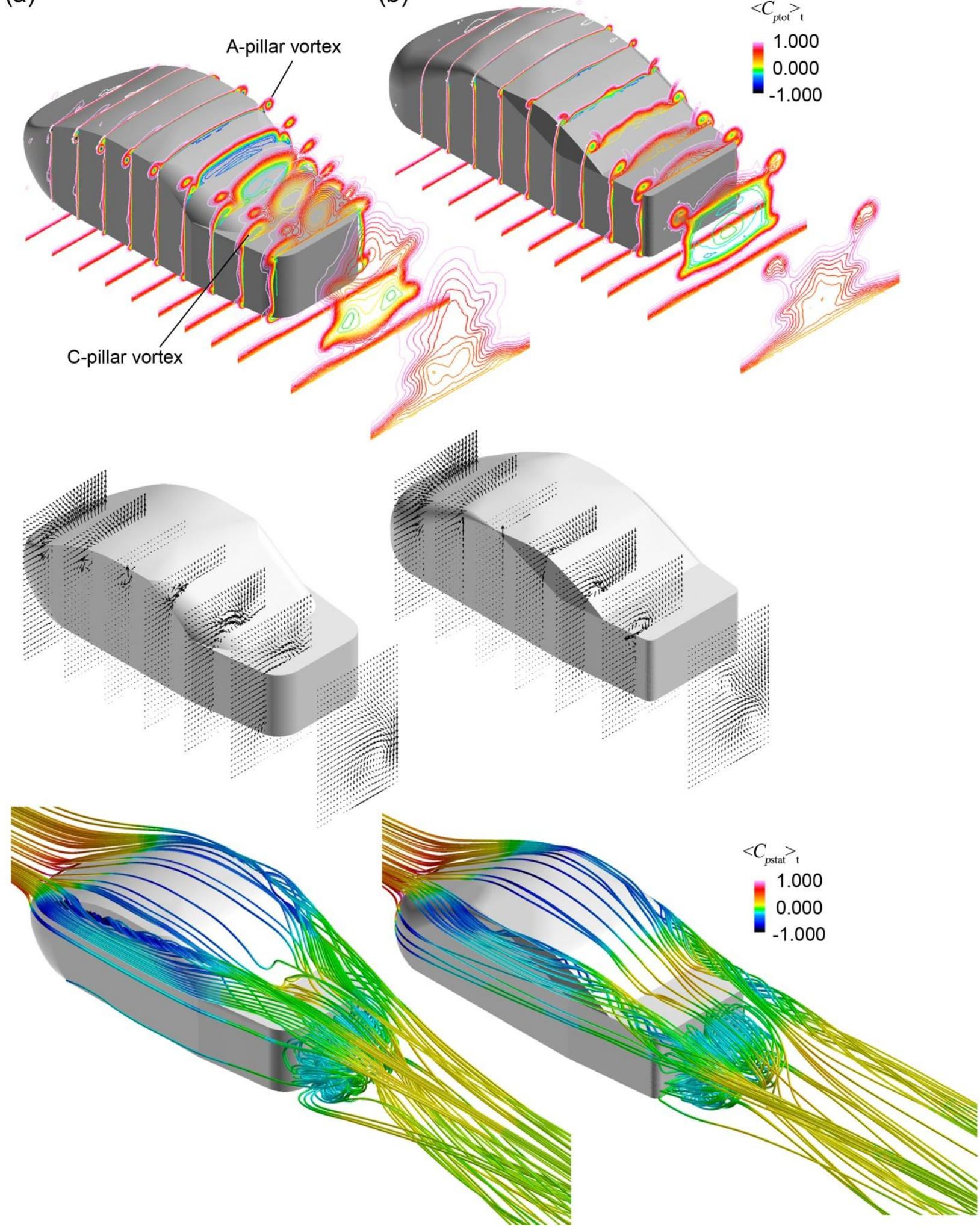

Fig. 11. Predominant flow structures around simplified vehicle models: (a) Model A; (b) Model B.

The flow regimes around the simplified models show a similar trend in an exaggerated fashion. That is, the A-pillar vortex appears only in model A, while the flow in the front section of model B is smooth. On the other hand, the C-pillar vortices generated in both simplified models appear stronger than the corresponding vortices generated by the real vehicles. Hence, it is inferred that the idealized notchback models successfully reproduce the predominant flow structures found on real vehicles with different pitching-stability characteristics. 


\subsection{Forced pitching oscillation}

\subsubsection{Periodic-pitching-oscillation condition}

Attributed to ride-height fluctuation, alteration in vehicle position can cause changes in the flow structures around the vehicle. Interaction between these flow structures and the vehicle body results in aerodynamic forces that can have the tendency to restore stability of the vehicle or to further enhance its motion. To probe the stability attitude of the models, a periodic pitching oscillation was imposed on them during LES by employing the ALE technique to rotate the models about a lateral axis. The axis is located at the lower part of the front section of the models at $0.821 l$ from the rear end, corresponding to the front-wheel axle of a real vehicle. This setting is in accordance with the road-test results of Okada et al. (2009), in which the notchbacks experienced more significant ride-height fluctuation at the back than the front. Hence, the models were rotated in a manner that simulates the rear-ride height fluctuation of the real vehicles. The pitch angle $\theta$ of the models is defined as $\theta=\theta_{0}+\theta_{1} \sin \varphi(t), \quad \varphi(t)=2 \pi f_{p} t$

By setting $\theta_{0}$ and $\theta_{1}$ equal to 2 , the vehicle models were forced to oscillate at an amplitude of $2^{\circ}$. Although this value is relatively larger than the range a vehicle would encounter under normal driving conditions, it has the advantage of producing more distinct aerodynamic damping effect in vehicles of different stability characteristics. Thus make it easier to interpret the underlying physical mechanism. Frequency $f_{p}$ was $10 \mathrm{~Hz}$, which corresponds to a Strouhal number $\left(\mathrm{S}_{\mathrm{t}}\right)$ of 0.13 , normalized by $l$ and $U_{\infty}$. This value was chosen in consideration of the $\mathrm{S}_{\mathrm{t}}$ value of 0.15 obtained by road test by Okada et al. (2009).

Figure 12 shows the sign convention for aerodynamic pitching moment and angle. The corresponding phase-averaged quantity was computed over 15 periods after the LES achieved a stable periodic condition. For each period, a total of 10,000 time steps was used. To cope with the high computing resources needed in the LES with ALE computation, the high-performance computing technique devised by Tsubokura et al. (2009a) was employed.

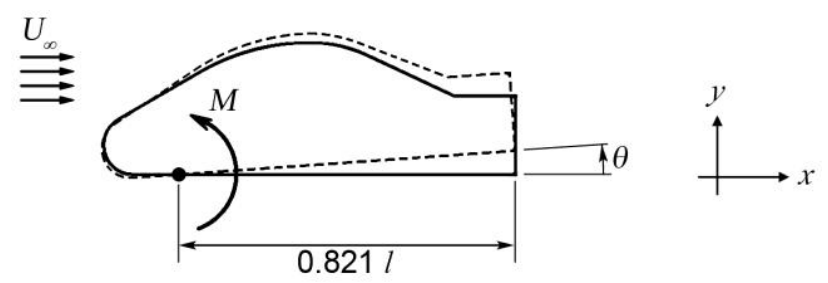

Fig. 12. Sign convention for $M$ and $\theta$.

\subsubsection{Dynamic effects on pitching moment due to model motion}

Figure 13 shows the time history of $M$ for models A and B. Attributed to vehicle-body motion, $M$ in the dynamic LES cases exhibits a nonlinear behavior. Figure 13(a) shows the time history of $M$ for model B obtained from a stationary simulation in which the model was fixed at $0^{\circ}$ pitch. The time series marches with stochastic fluctuation as anticipated by transient simulations of flow with turbulent nature. In the dynamic LES, depicted in Fig. 13(b), the time history exhibits both periodic and stochastic fluctuations. Figure 14 illustrates these quantities, including the time-averaged and phase-averaged values. (Note that the instantaneous quantities, $M=M^{\prime}+\langle M\rangle_{t}$, range from 0.6 to $0.7 \mathrm{~s}$; model B). To extract meaningful information from the results, phase-averaging of $M$ was performed. As for the stationary LES simulation results, it was time-averaged over $0.3 \mathrm{~s}$ physical time. 
(a)
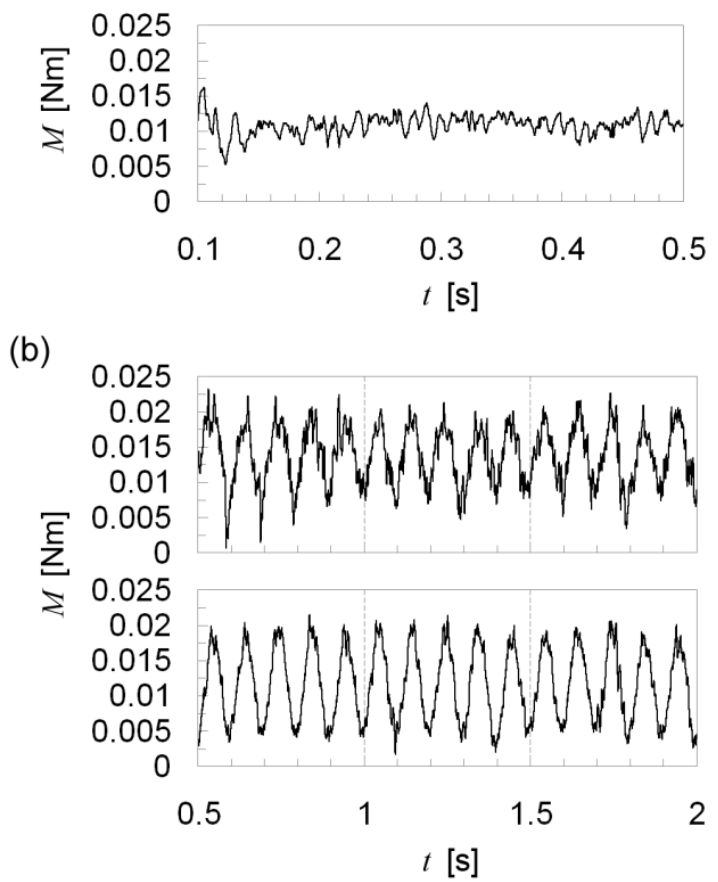

Fig. 13. Time history of M: (a) Stationary LES; Model B; (b) Dynamic LES; Model A (above); Model B (below).

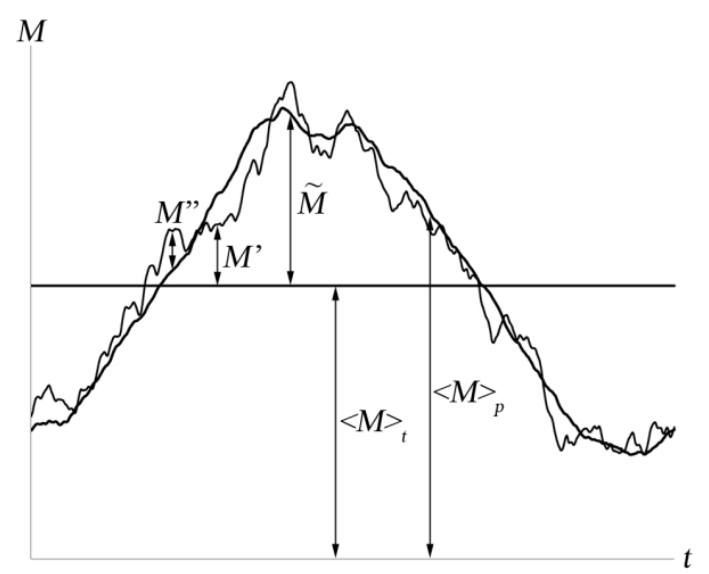

Fig. 14. Periodic and stochastic fluctuations in $M$ obtained from dynamic LES. $\tilde{M}$ periodic fluctuation; $M^{\prime \prime}$ stochastic turbulent fluctuation; $M^{\prime}$ total fluctuation; $\langle M\rangle_{p}$ phase-averaged value; $\langle M\rangle_{t}$ time-averaged value.

Figure 15 shows $\langle M\rangle_{p}$ as a function of phase angle for models A and B. In general, the shapes of the curves (a) and (b) are similar; namely, $\langle M\rangle_{p}$ reaches the minimum peak during tail-up motion and the maximum peak during tail-down motion. Hence, the two models are aerodynamically stable with respect to pitching oscillation. Conversely, the phase shift of the curves is slightly different. In addition, model B has a wider fluctuation range owing to the lower minimum peak.

For a pitch angle of $2^{\circ}$ (i.e., at $0^{\circ}$ and $180^{\circ}$ phases) in Figures 15(a) and (b), the dynamic LES results for the tail-up and tail-down pitching phases show totally different behavior. This is because the airflow pattern varies with the different vehicle-body motions of the two models. To compare the results with the quasi-steady prediction, the LES was also conducted under the stationary condition with the models fixed at $0^{\circ}, 2^{\circ}$, and $4^{\circ}$ pitch. Figure 15 shows that the corresponding quasi-steady results, i.e. $\langle M\rangle_{t}$, failed to capture the dynamically obtained tendency. In general, the quasi-steady predictions overestimate and underestimate the dynamic values of $M$ at phase angles of $0^{\circ}$ and $180^{\circ}$ by, respectively, about $23 \%$ and $41 \%$ in the case of model $\mathrm{A}$ and by about $56 \%$ and $54 \%$ in the case of model B. Moreover, at any given pitch angle, only one corresponding $\langle M\rangle_{t}$ can be obtained by the quasi-steady approach. This result indicates that, despite the same momentary body position, it is not feasible to approach the dynamic problems with static methods in a situation when the type of vehicle motion is an influential factor. 

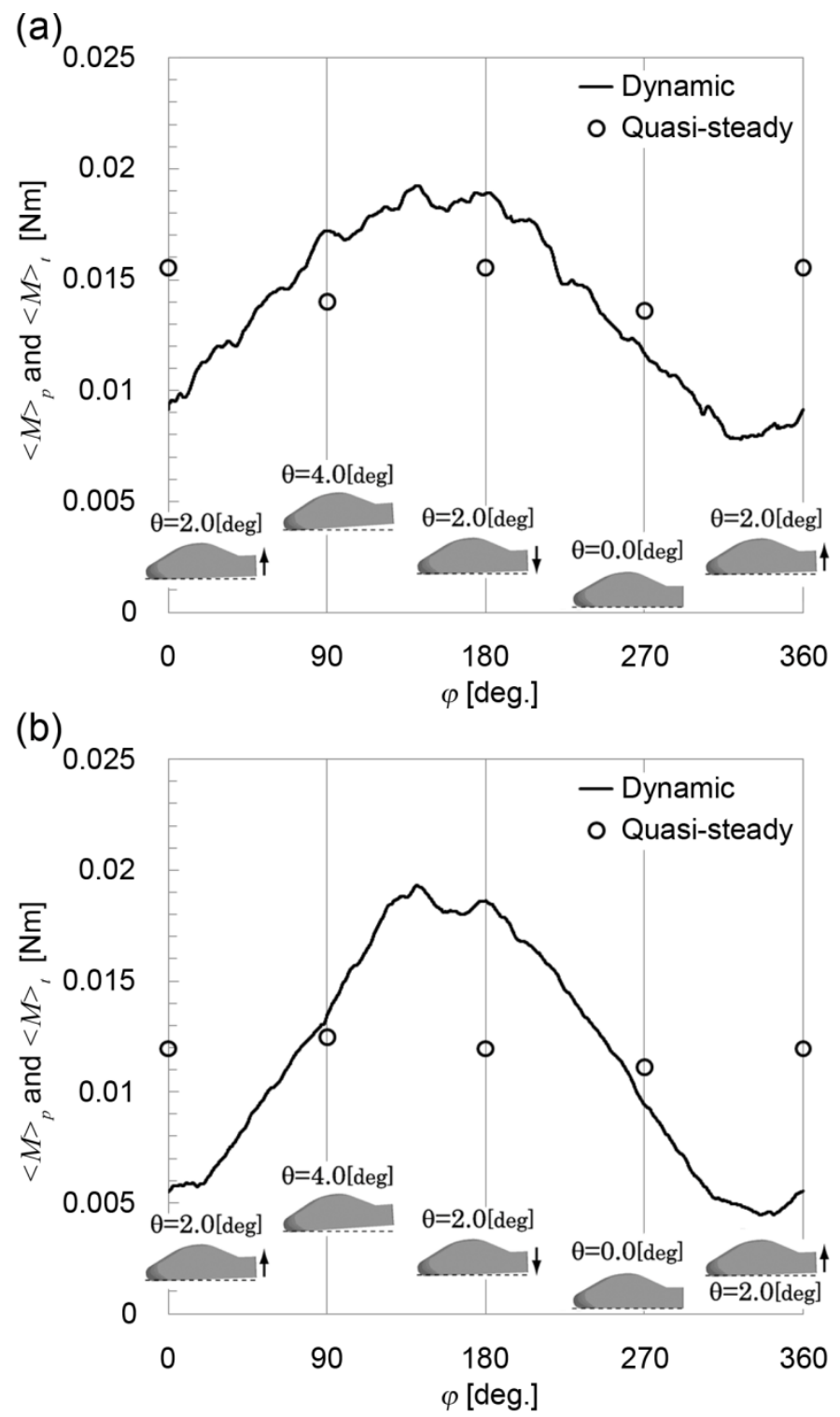

Fig. 15. Comparison between quasi-steady and dynamic LES estimations: (a) Model A; (b) Model B.

\subsubsection{Formulation of aerodynamic-damping coefficient}

To assess the dynamic responses of the models under the influence of pitching oscillation, the resulting $\langle M\rangle_{p}$ from dynamic LES is decomposed into steady and unsteady components. The equation for $\langle M\rangle_{p}$ in terms of $\theta$ is given as the following expansion,

$$
<M>_{p}=C_{0}+C_{1} \theta+C_{2} \dot{\theta}+C_{3} \ddot{\theta} \text {, }
$$

where, respectively, the single dot and double dots in the third and fourth terms indicate the first and second derivatives with respect to $t$. Both $C_{0}$ and $C_{1}$ are static components; the former denotes the pitching moment at zero pitch, while the latter describes the quasi-static behavior by taking into account the pitch-angle variation in a static manner. $C_{2}$ is associated with aerodynamic damping, and $C_{3}$ is an added moment of inertia that is proportional to angular acceleration.

Substituting eq. (12) into (13) and rearranging gives

$$
<M>_{p}=\left(C_{0}+C_{1} \theta_{0}\right)+\left[C_{1}-\left(2 \pi f_{p}\right)^{2} C_{3}\right] \theta_{1} \sin \varphi(t)+2 \pi f_{p} \theta_{1} C_{2} \cos \varphi(t) \text {. }
$$

The above equation can then be rewritten by using new parameters, namely, $M_{\text {stat }}, M_{\text {sin }}$ and $M_{\cos }$ as $<M>_{p}=M_{\text {stat }}+M_{\text {sin }} \sin \varphi(t)+M_{\text {cos }} \cos \varphi(t)$.

These parameters can be presented in a non-dimensional form. If they are normalized in a similar manner to the pitching-moment coefficient, the parameters become 


$$
C_{\text {stat }}=\frac{M_{\text {stat }}}{1 / 2 \rho U_{\infty}^{2} A l_{w}}, C_{\text {sin }}=\frac{M_{\text {sin }}}{1 / 2 \rho U_{\infty}^{2} A l_{w}}, C_{\text {cos }}=\frac{M_{\text {cos }}}{1 / 2 \rho U_{\infty}^{2} A l_{w}},
$$

where $\rho, U_{\infty}, A$, and $l_{w}$ are fluid density, mainstream velocity, vehicle frontal area, and wheelbase. Here, $C_{\text {stat }}$ is a constant, and $C_{\text {sin }}$ is in-phase with the imposed displacement. In the case of periodic pitching oscillation, these two components produce zero net work on the model over a full cycle of oscillation. On the contrary, $C_{\text {cos }}$ that is inphase with the angular velocity, is the component that produces work on the model. Hence, this parameter reflects the dynamic response of the vehicle. It depends on the sign of $C_{\mathrm{cos}}$, a negative value implies a tendency for aerodynamics to damp the pitching oscillation, whereas a positive value enhances the vehicle motion. The coefficient thus enables quantitative evaluation of vehicle stability; therefore, it is termed "aerodynamic-damping coefficient."

\subsubsection{Comparison of two aerodynamic configurations}

The coefficients in Eq. (16) are obtained by fitting the phase-averaged pitching moment to Eq. (15) by nonlinear least squares regression. Figure 16 shows the curves of phase-averaged aerodynamic pitching moment and the corresponding fitted functions for model A and model B. Table 3 summarizes the obtained coefficients for comparison. As shown in the table, the $C_{\mathrm{cos}}$ of the two models are negative, implying a tendency to resist the pitching motion of the models. This result again confirms that both models are aerodynamically stable, as discussed in section 3.2.2. Between them, however, model B has a higher aerodynamic-damping coefficient, by about $40.81 \%$. This finding is consistent with the fact that model B was created on the basis of a real vehicle with higher stability.

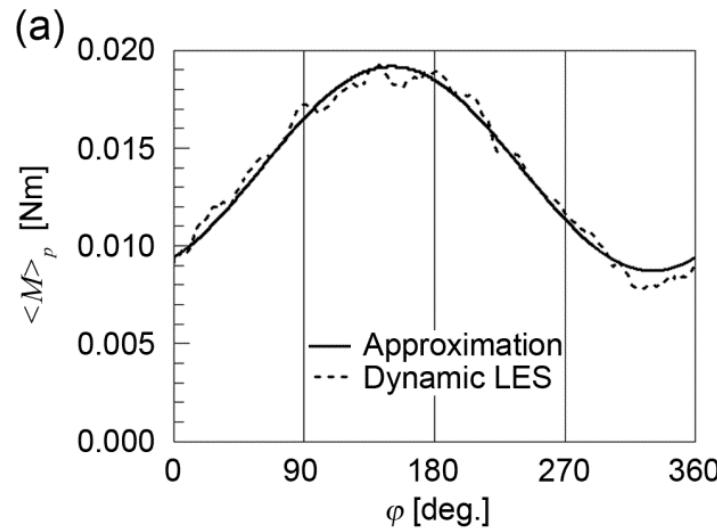

(b)

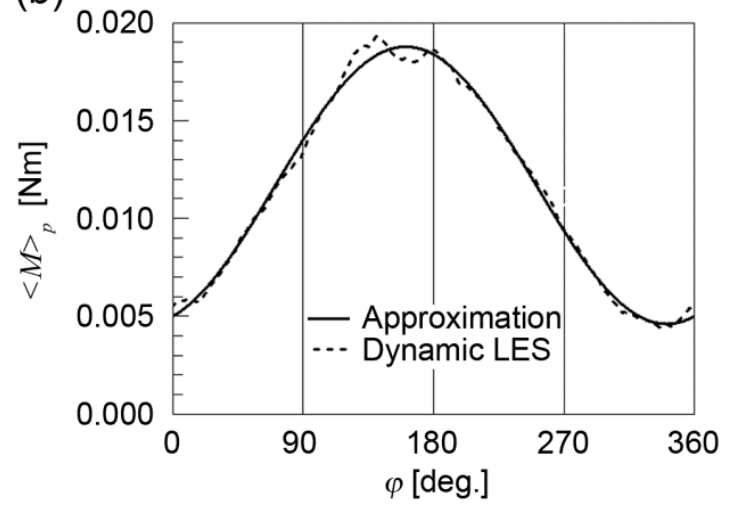

Fig. 16. Phase-averaged aerodynamic pitching moment and fitted functions: (a) Model A; (b) Model B.

Table 3. Coefficients for aerodynamic pitching moment approximation function.

$\begin{array}{llll}\text { Model } & C_{\text {stat }} & C_{\text {sin }} & C_{\text {cos }} \\ \text { A } & 0.12 & 0.02 & -0.040 \\ \text { B } & 0.10 & 0.02 & -0.059\end{array}$

Table 4 summarizes the proportional contributions of each vehicle-body part (shown in Fig. 17) on the aerodynamic-damping coefficient. On average, the underbody has the highest contribution (up to $78.62 \%$ and $61.11 \%$ of the total in model A and model B, respectively), followed by the roof and trunk deck. This result is not surprising for two reasons: the underbody and roof have larger surface area and moment arm; and most of the 
predominate flow structures are generated above the trunk deck. The induced pressure force as a result of interaction between the transient flow structures and the three body parts, therefore, produces a large amount of transient pitching moment that contributes to $C_{\mathrm{cos}}$. However, the difference in the contributions of the underbody and roof for the two models was only $12 \%$ and $9 \%$, while the difference between the trunk-deck contributions is considerable, namely, $131 \%$. Hence, the trunk-deck contribution accounts for the different pitching-stability characteristics obtained with the two models. For the details of the aerodynamic-damping mechanism, interested readers are referred to Cheng et al. (2011).

It should be noted that the fixed wall-boundary condition used for the ground was used to model the fixedfloor wind-tunnel experimental environment. A more realistic treatment to model the situation when a vehicle is running on the road is to apply the velocity-wall boundary condition set at the vehicle's speed. The proportion contribution of the under body on aerodynamic damping may therefore vary when a different boundary condition is adopted owing to the effect of ground. Moreover, when it is attempted to model the damping of a real vehicle, a higher Re range (typically of the order of six), a realistic pitch-angle variation, and the effect of ambient turbulence should be considered.

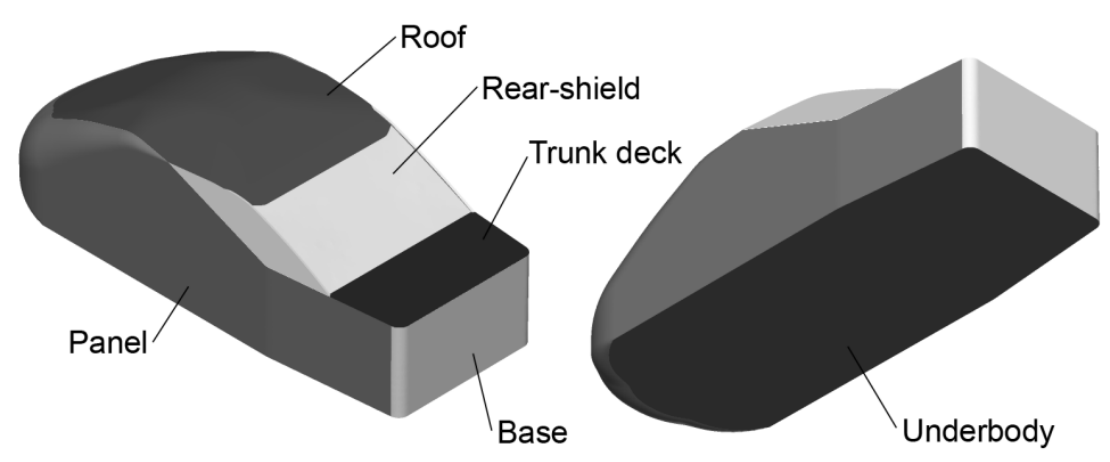

Fig. 17. Body-part designations.

Table 4. Contribution of body part on aerodynamic damping coefficient $C_{\cos }$.

$\begin{array}{lllll}\text { Body Part } & \text { Model A } & (\%) & \text { Model B } & (\%) \\ \text { Underbody } & -0.032 & (78.62) & -0.036 & (61.11) \\ \text { Roof } & -0.0068 & (16.71) & -0.0062 & (10.52) \\ \text { Trunk deck } & -0.0021 & (5.16) & -0.010 & (16.98) \\ \text { Rear shield } & -0.00036 & (0.88) & -0.0073 & (12.39) \\ \text { Base } & 0.00017 & (-0.42) & 0.00013 & (-0.22) \\ \text { Panel } & 0.00039 & (-0.96) & 0.00046 & (-0.78)\end{array}$

\section{Concluding remarks}

Taking into account the effect of transient aerodynamics, a new index - termed "aerodynamic-damping coefficient" - is introduced for automotive application. It enables quantitative evaluation of vehicle stability. Hence, when used in conjunction with the commonly applied drag and lift coefficients, it can improve realism in assessment of vehicle aerodynamics. For an idealized notchback model with a simple bluff body shape, the index showed that the underbody contribution alone accounts for about $69 \%$ of the total aerodynamic damping with respect to pitching oscillation. For a more comprehensive understanding of their effect on vehicle stability, therefore, various underbody configurations should be further investigated. Particularly, it is necessary to determine the underlying mechanism by which transient aerodynamics can be exploited for damping of pitching oscillation. While the example presented in this study is based on pitching stability, the method can be extended to include other aerodynamic properties like yawing and rolling. In addition, further work is required to investigate the dependency of $C_{\cos }$ on Re, St, and oscillation amplitude.

\section{Acknowledgments}

This work was supported by the 2007 Industrial Technology Research Grant program from the New Energy and Industrial Technology Development Organization (NEDO) of Japan. Development of the base software FFR was supported by the FSIS and "Revolutionary Simulation Software (RSS21)" projects sponsored by MEXT, Japan. 


\section{References}

Amsden, A. A., Harlow, F. H., 1970. A simplified MAC technique for incompressible fluid flow calculations. Journal of Computational Physics. 6, 322-5.

Aschwanden, P., Müller, J., Knörnschild, U., 2006. Experimental study on the influence of model motion on the aerodynamic performance of a race car. SAE Paper 2006-01-0803.

Aschwanden, P., Müller, J., Travaglio, G. C., Schöning, T., 2008. The Influence of Motion Aerodynamics on the Simulation of Vehicle Dynamics. SAE Paper 2008-01-0657.

Baker, C.J., 1991a. Ground vehicles in high cross winds-Part I Steady aerodynamic forces. Journal of Fluids and Structures. 5, 69-90.

Baker, C.J., 1991b. Ground vehicles in high cross winds-Part 2 Unsteady aerodynamic forces. Journal of Fluids and Structures. 5, 91-111.

Baker, C.J., 1991c. Ground vehicles in high cross winds-Part 3 The interaction of aerodynamic forces and the vehicle system. Journal of Fluids and Structures. 5, 221-241.

Baker, C., Reynolds, S., 1992. Wind induced accidents of road vehicles. Accident Analysis \& Prevention. 24, 559575.

Sigbjörnsson, R., Snæbjörnsson, J.T., 1998. Probabilistic assessment of wind related accidents of road vehicles: a reliability approach. Journal of Wind Engineering and Industrial Aerodynamics. 74 (6), 1079-1090.

Baker, C., Cheli, F., Orellano, A., Paradot, N., Proppe, C., Rocchi, D., 2009. Cross-wind effects on road and rail vehicles. Vehicle System Dynamics. 47, 983-1022.

Buchheim, R., Leie, B., Luckoff, H.J., 1983. Der neue Audi 100- Ein Beispiel fur Konsequente Aerodynamische Personenwagen-Entwicklung. ATZ 85, 419-425.

Charuvisit, S., Kimura, K., Fujino, Y., 2004. Experimental and semi-analytical studies on the aerodynamic forces acting on a vehicle passing through the wake of a bridge tower in cross wind. Journal of Wind Engineering and Industrial Aerodynamics. 92 (9), 749-780.

Cheng, S.Y., Tsubokura, M., Nakashima, T., Nouzawa, T., Okada, Y., 2011. A numerical analysis of transient flow past road vehicles subjected to pitching oscillation. Journal of Wind Engineering and Industrial Aerodynamics. 99, 511-522.

Dominy, R.G., Ryan, A., Sims-Williams, D.B., 2000. The aerodynamic stability of a Le Mans prototype race car under off-design pitch conditions. SAE Paper 2000-01-0872.

Dotson, K.W., Engblom, W.A., 2004. Vortex induced vibration of a heavy-lift launch vehicle during transonic flight. Journal of Fluids and Structures. 19, 669-680.

Guilmineau, E., Chometon, F., 2008. Numerical and Experimental Analysis of Unsteady Separated Flow behind an Oscillating Car Model. SAE Paper 2008-01-0738.

Hirt, C.W., Amsden, A.A., Cook, J.L., 1974. An Arbitrary Lagrangian-Eulerian Computing Method for All Flow Speeds, Journal of Computational Physics. 14, 227-253.

Hucho, W-H., Sovran, G., 1993. Aerodynamics of road vehicles. Annual Review of Fluid Mechanics. 25, $485-537$. Hunt, J. C. R., Wray, A. A., Moin, P., 1988. Eddies, stream, and convergence zones in turbulent flows. Technical Report. CTR-S88. Center for Turbulence Research, Stanford University.

Katz, J., 2006. Aerodynamics of Race Cars. Annual Review of Fluid Mechanics. 38, 27-63.

Kleissl, K., Georgakis, C. T., 2011. Aerodynamic control of bridge cables through shape modification: A preliminary study. Journal of Fluids and Structures. 27 (7), 1006-1020.

Macdonald, J. H. G., Larose, G. L., 2006. A unified approach to aerodynamic damping and drag/lift instabilities, and its application to dry inclined cable galloping. Journal of Fluids and Structures. 22 (2), 229-252.

Macklin, R., Garry, K., Howell, J., 1997. Assessing the effects of shear and turbulence during the dynamic testing of the crosswind sensitivity of road vehicles. SAE Paper 970135.

Mansor, S., Passmore, M. A., 2008. Estimation of bluff body transient aerodynamics using an oscillating model rig. Journal of Wind Engineering and Industrial Aerodynamics. 96, 1218-1231.

Noger, C., Regardin, C., Szechenyi, E., 2005. Investigation of the transient aerodynamic phenomena associated with passing manoeuvres. Journal of Fluids and Structures. 21 (3), 231-241.

Okada, Y., Nouzawa, T., Nakamura, T., Okamoto, S., 2009. Flow structure above the trunk deck of sedan-type vehicles and their influence on high-speed vehicle stability 1st report: On-Road and Wind-Tunnel Studies on Unsteady Flow Characteristics that Stabilize Vehicle Behavior. SAE Paper No 2009-01-0004.

Païdoussis, M. P., 2006. Real-Life Experiences With Flow-Induced Vibration. Journal of Fluids and Structures. 22 (6-7), 741-755. 
Passmore, M. A., Richardson, S., Imam, A., 2001. An experimental study of unsteady vehicle aerodynamics.

Proceedings of the Institution of Mechanical Engineers, Part D: Journal of Automobile Engineering. 215 (7), 779 788.

Poirel, D., Métivier, V., Dumas, G., 2011. Computational aeroelastic simulations of self-sustained pitch oscillations of a NACA0012 at transitional Reynolds numbers. Journal of Fluids and Structures. 27 (8), 1262-1277.

Roache, P.J., 1998. Verification and Validation in Computational Science and Engineering, Hermosa Publishers, Albuquerque, New Mexico.

Ryan, A., Dominy, R.G., 1998. The aerodynamic forces induced on a passenger vehicle in response to a transient cross-wind gust at a relative incidence of 30md. SAE Paper 980392.

Smagorinsky, J., 1963. General circulation experiments with primitive equations. Monthly Weather Review. 91-3, 99-164.

Tsubokura, M., Nakashima, T., Kitoh, K., Sasaki, Y., Oshima, N., Kobayashi, T., 2009a. Development of an Unsteady Aerodynamic Simulator Using Large-Eddy Simulation Based on High-Performance Computing Technique. SAE International Journal of Passenger Cars; Mechanical Systems. 2-1, 168-178.

Tsubokura, M., Kobayashi, T., Nakashima, T., Nouzawa, T., Nakamura, T., Zhang, H., Onishi, K., Oshima, N., 2009b. Computational visualization of unsteady flow around vehicles using high performance computing.

Computers \& Fluids. 38, 981-990.

Tsubokura, M., Nakashima, T., Kitayama, M., Ikawa, Y., Deog, H. D., Kobayashi, T., 2010. Large eddy simulation on the unsteady aerodynamic response of a road vehicle in transient crosswinds. International Journal of Heat and Fluid Flow. 31, 1075-1086.

\section{Nomenclature}

$C_{\cos } \quad$ coefficient of aerodynamic damping

$C_{d} \quad$ coefficient of drag

$C_{p \text { tot }} \quad$ coefficient of total pressure, $C_{p \text { tot }}=\left(1 / 2 \rho U^{2}+p\right) /\left(1 / 2 \rho U_{\infty}^{2}\right)$

$C_{p \text { stat }}$ coefficient of static pressure, $C_{p \text { stat }}=\left(p-p_{\infty}\right) /\left(1 / 2 \rho U_{\infty}^{2}\right)$

$C_{s} \quad$ Smagorinsky coefficient

$D \quad$ aerodynamic drag

$f \quad$ instantaneous value of a quantity

$\bar{f} \quad$ filtered value of $f$

$<f>_{t}$ time-averaged value of $f$

$<f>_{p} \quad$ phase-averaged value of $f$

$\tilde{f} \quad$ periodic fluctuations, $\left.\tilde{f}=<f>_{p}-<f\right\rangle_{t}$

$f^{\prime \prime} \quad$ stochastic turbulent fluctuations, $f^{\prime \prime}=f-<f>_{p}$

$f^{\prime} \quad$ total fluctuations, $\left.f^{\prime}=f-<f\right\rangle_{t}$

$f_{d} \quad$ Van Driest function

$f_{p} \quad$ frequency of pitching oscillating of vehicles

Fs safety factor

GCI error band

$h \quad$ vehicle height

$L \quad$ aerodynamic lift

$l \quad$ vehicle length

M aerodynamic pitching moment

$\boldsymbol{n}_{s} \quad$ outward unit normal vector to control volume surface

$p \quad$ static pressure 
$p_{\infty} \quad$ static pressure at the inlet boundary

$r \quad$ refinement ratio

$\operatorname{Re} \quad$ Reynolds number, $\operatorname{Re}=U l / v$

$S \quad$ surface of control volume

$S_{i j} \quad$ strain rate tensor

St $\quad$ Strouhal number, $\mathrm{St}=f_{p} l / U_{\infty}$

$t$ time

$\boldsymbol{u}_{d} \quad$ deformation velocity vector of control volume surface center

$u_{g} \quad$ deformation speed of control volume surface

$u_{i} \quad$ three component of velocity vector $(i=1,2,3$ : streamwise, lateral, spanwise $)$

$U_{\infty} \quad$ mainstream velocity

$w \quad$ vehicle width

$x_{i} \quad$ three component of spatial coordinate $(i=1,2,3$ : streamwise, lateral, spanwise)

$y^{+} \quad$ non-dimensional wall distance

$\gamma \quad$ order of convergence

$\Delta \quad$ length scale of subgrid-scale turbulence

$\Delta t \quad$ time difference

$\theta$ pitch angle

$v \quad$ kinematic viscosity

$v_{\mathrm{SGS}} \quad$ subgrid-scale eddy viscosity

$\rho \quad$ fluid density

$\varphi \quad$ phase angle

$\Omega \quad$ volume of control volume 